\title{
Finger Photoplethysmography to Monitor Chest Compression Rate During Out-of-Hospital Cardiac Arrest
}

\author{
Andoni Elola ${ }^{1}$, Jon Urteaga ${ }^{1}$, Elisabete Aramendi ${ }^{1}$, Unai Irusta ${ }^{1}$, Erik Alonso ${ }^{1}$, \\ Mohamud Daya $^{2}$, Pamela Owens ${ }^{3}$, Ahamed Idris ${ }^{3}$ \\ ${ }^{1}$ University of the Basque Country, Bilbao, Spain \\ ${ }^{2}$ Oregon Health \& Science University, Portland, OR, USA \\ ${ }^{3}$ University of Texas SouthWestern Medical Center, TX, USA
}

\begin{abstract}
Cardiac arrest survival rate is strongly associated with high quality cardiopulmonary resuscitation (CPR), which includes chest compression (CC) rates above $100 \mathrm{~min}^{-1}$. Currently, defibrillator monitors use external hardware such as CPR assist pads to monitor CC rate and give feedback to the rescuer. The photoplethysmogram (PPG) provides information about the level of oxygen saturation in blood and can be easily recorded by a pulse oximeter in the fingertip. The aim of this study was to analyze the feasibility of using the finger PPG to monitor the presence and rate of $\mathrm{CCs}$ in out-of-hospital cardiac arrest (OHCA). The dataset used in the study consisted of 112 segments from 46 OHCA patients, with a total duration of $256 \mathrm{~min}$ and 27667 CCs. The method is based on the power spectral density analysis of $10 \mathrm{~s}$ segments of the PPG. CC presence was determined through thresholding, and $C C$ rate was computed applying a maximum slope criterion. The dataset was divided patient-wise intro training (60\%) and testing (40\%) sets. For the test set the algorithm presented a sensitivity and a positive predictive value of $85.2 \%$ and $98.1 \%$ respectively for $C C$ detection, a CC rate error of $2.8(6.8) \mathrm{min}^{-1}$ and $3.4 \%$ of the values with an error above $10 \%$.
\end{abstract}

\section{Introduction}

Cardiac arrest is the sudden and unexpected loss of heart function, which if not treated may lead the patient to sudden cardiac death (SCD). SCD is the main cause of death in the industrialized world with an incidence of 38 cases per 100000 person-year in Europe [1]. Early defibrillation and cardiopulmonary resuscitation (CPR), consisting of chest compressions (CCs) and ventilations, permit restoring spontaneous circulation. The latest European Resuscitation Council (ERC) guidelines recommend CCs with a rate of 100-120 $\mathrm{min}^{-1}$ and a minimum depth of $5 \mathrm{~cm}$ [2].
Some comertial defibrillators are equipped with additional devices that include accelerometers and force sensors to monitor rate and depth of CCs, which are then used to provide real time feedback to rescuers providing CPR [3-6].

The photopletismogram (PPG) signal acquired by fingertip sensors estimates the arterial oxygen saturation of the patient $[7,8]$ and is widely used to monitor hemodinamically stable patients. For instance, pulse or respiration rates are frequently monitored using the PPG in stress tests [9].

Due to its simplicity and low cost, the PPG signal has been used in different applications. Recently new applications of PPG signal have been proposed in out-of-hospital cardiac arrest (OHCA) $[10,11]$. This study analyzes the feasibility of the PPG to accurately provide information on the presence and rate of CCs during OHCA.

\section{Materials}

The dataset used in this study is a subset of a large database acquired from OHCA patients. The episodes were recorded by the DFW Center for Resuscitation Research (UTSW, Dallas) and the Clackamas County Fire District \# 1 (Clackamas, Oregon).

The electronic files recorded by Zoll E-Series defibrillators were collected from 46 patients. A total of $112 \mathrm{seg}$ ments were extracted with concurrent PPG and the CCwave signal acquired by the CPR-padz feedack device. The CC-depth signal was also available with the instants and depths of every CC detected by the CPR-padz. The segments included at least $60 \mathrm{~s}$ of CCs with gaps of maximum $10 \mathrm{~s}$.

Figure 1 shows an example of a raw PPG segment (top panel) and the CC-depth signal (bottom panel) that provides information about the depth and the instants of CCs. 


\section{Methods}

\subsection{Preprocessing and filtering}

The PPG signal was preprocessed firstly with an interpolation filter (cubic spline) to fill the gaps presented in the raw signal. Then it was band-pass filtered between 1$3.2 \mathrm{~Hz}$ using an order 3 Butterworth filter to removed the baseline drift and low and high frequency noise. Figure 1 also shows the preprocessed PPG and the band-pass filtered signal.

\section{2. $\quad \mathrm{CC}$ presence detection}

The preprocessed PPG signal was windowed using 10 s Kaiser $(\beta=3)$ window with $50 \%$ of overlap. Then, the power spectral density (PED) was calculated as the square of the module of the 4096-point Fast Fourier Transform. First the main frequency of the compressions, $f_{\mathrm{cc}}$, was identified as the frequency correspondig to the PED peak for which the lobe around the peak presented the highest value. If the power around $f_{\mathrm{cc}}$ was higher than a given percentage, $P_{\mathrm{th}}$, the segment was labelled as a CC segment.

Figure 2 shows a $10 \mathrm{~s}$ window of the PPG and the PED in terms of $\mathrm{CC}$ rate $\left(\mathrm{min}^{-1}\right)$.

\section{3. $\quad \mathrm{CC}$ rate calculation}

For segments labelled as CC segments, the rate of the compressions was considered to be $f_{\text {cc }}$, the main frequency on the PED. Figure 2 shows a $10 \mathrm{~s}$ window of the filtered PPG, the CC-wave and the PED in terms of CC rate $\left(\mathrm{min}^{-1}\right)$. The dashed green line in the PED represents $f_{\mathrm{cc}}$, while the dashed red line depicts $f_{\mathrm{g}}$, the $\mathrm{CC}$ rate computed from the instants, $t_{\mathrm{i}}$, marked on the $\mathrm{CC}$-wave. $f_{\mathrm{g}}$ was computed as the inverse of the median time intervals between compressions $\left(t_{\mathrm{i}+1}-t_{\mathrm{i}}\right)$ and it was used as gold standard to evaluate the accuracy of the algorithm based on the PPG.
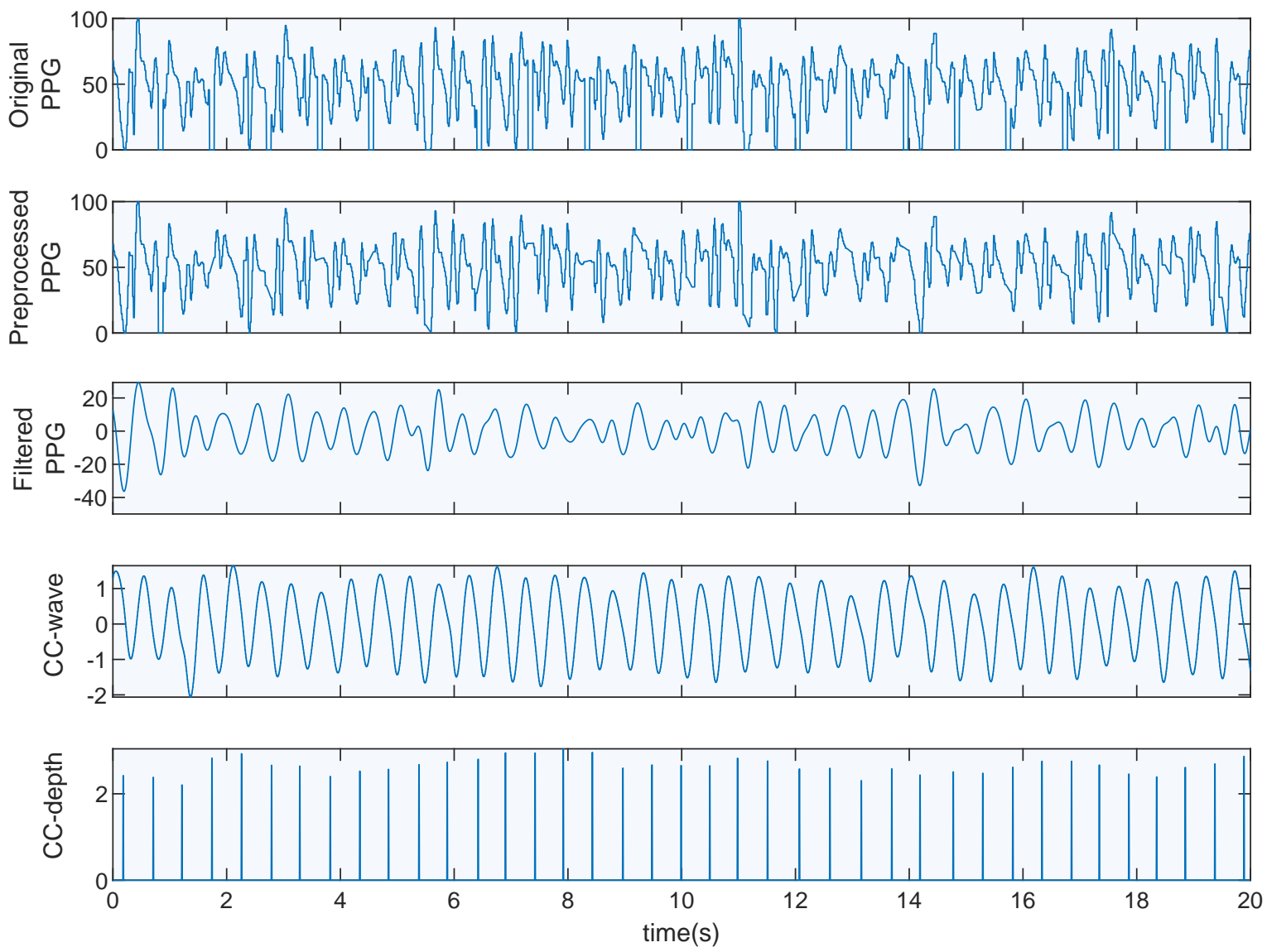

Figure 1. An example of a segment of the dataset of the study. From top to bottom: the segment of the original, preprocessed and filtered PPG; the signal provided by the CPR-padz, the CC-wave and the CC-depth, used as gold standard. 

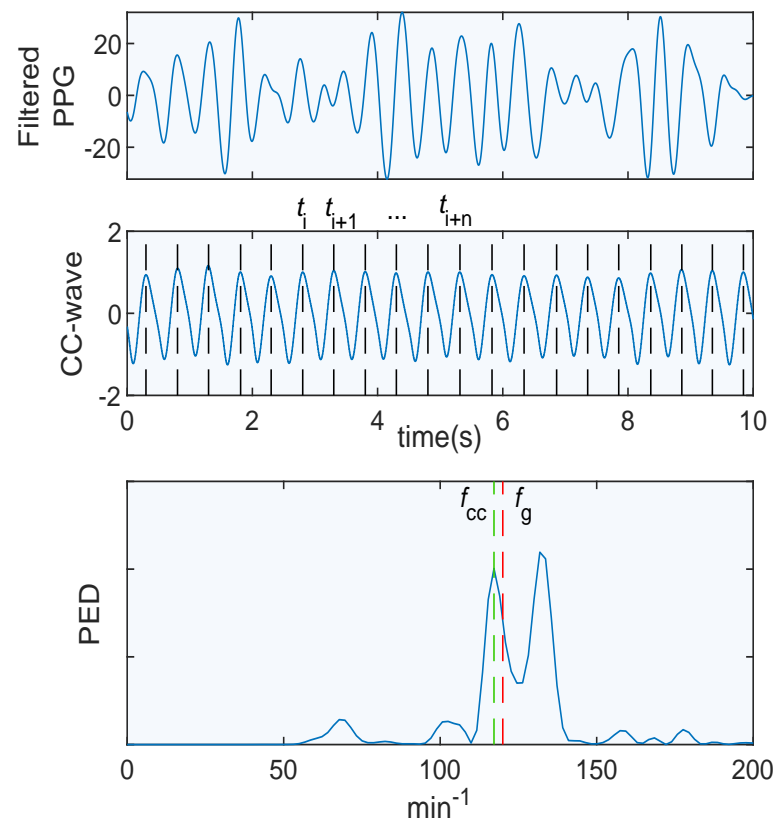

Figure 2. A $10 \mathrm{~s}$ window of the filtered PPG, the CCwave signal and the PED used to compute the presence and rate of CCs. The instants of CCs, $t_{i}$, are depicted as vertical lines on the $\mathrm{CC}$-wave signal. The peak of the PED was identified as the $\mathrm{CC}$ rate, $f_{\mathrm{cc}}$, and compared the rate obtained from the gold standard, $f_{\mathrm{g}}$.

\subsection{Statistical evaluation of the algorithm performance}

CC presence was evaluated in terms of sensitivity (Se), proportion of windows with CCs correctly identified, and positive predictive value (PPV), proportion of windows identified with CCs that truly had CCs. A segment was labelled as $\mathrm{CC}$ segment if the more than 10 compressions were present in the $10 \mathrm{~s}$ interval.

The accuracy of the algorithm computing the $\mathrm{CC}$ rate was evaluated by comparing $f_{\mathrm{cc}}$ and $f_{\mathrm{g}}$. The mean (standard deviation, SD) of the absolute error and the percentage of errors above $10 \%\left(\mathrm{Pe}_{10}\right)$ were calculated. The Bland-Altman plot for the error for the complete dataset and the error per patient were computed.

Data were randomly split patient-wise into training/test sets $(60 / 40 \%)$, to optimize the $P_{\text {th }}$ and validate the method, respectively. This procedure was repeated 50 times to obtain statistically meaningful results.

\section{Results}

The dataset containing 112 segments with a total duration of $256 \mathrm{~min}$ and $27667 \mathrm{CCs}$ were analyzed. The mean (SD) duration of the segments was 137 (115) s with 247 (213) CCs per segment. A total of 2907 windows

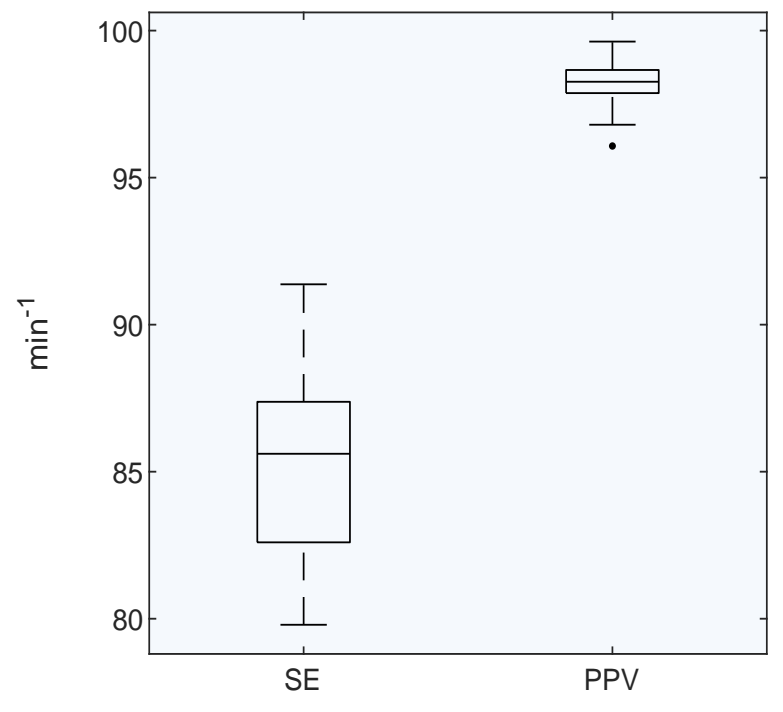

Figure 3. Sensitivity and positive predictive value per replica for the test set. The box shows the median and interquartile range (IQR), and the whiskers the last values within $\pm 1.5 \mathrm{IQR}$

\section{(84.4\% with CCs) were processed.}

The average performance of the algorithm in terms of mean (SD) in the training dataset was $\mathrm{Se}=87.6(2.1) \%$, $\mathrm{PPV}=98.1(0.4) \%$, absolute $\mathrm{CC}$ rate error of 2.4(0.3) $\min ^{-1}$ and $P e_{10}=2.4(0.8) \%$. For the test set the average performance was $\mathrm{Se}=85.2(3.1) \%, \mathrm{PPV}=98.1(0.7) \%$, absolute CC rate of $2.8(0.5) \mathrm{min}^{-1}$ and $P e_{10}=3.4(1.4) \%$.

Figure 3 shows the performance of $\mathrm{CC}$ rate calculation. The box plots represents the sensitivity and positive predictive value for the 50 repetitions for the test set.

Figure 4 shows the Bland-Altman plot for the error $f_{\mathrm{cc}^{-}}$ $f_{\mathrm{g}}$ for one replica with $P_{\mathrm{th}}=26$. The black line shows the mean error, $1.39 \mathrm{~min}^{-1}$, and the dashed black lines show the $95 \%$ level of agreement.

\section{Discussion and conclusions}

Monitoring the quality of CPR is crucial to adhere the rate of $\mathrm{CCs}$ to the range recommended by resuscitation guidelines. Current defibrillators require additional hardware with accelerometers or force sensors to evaluate the $\mathrm{CC}$ rate. The PPG signal is a commonly used to estimate the arterial oxygen saturation of the patient, and it can be easily acquired by simple fingertip sensors. This study evaluates the feasibility of using the PPG to detect the presence and rate of CCs in OHCA.

The proposed method was tested with segments of PPG signals including CCs. The algorithm processed windows of $10 \mathrm{~s}$ and provided a Se/PPV of $85 / 98 \%$ and a mean CC 


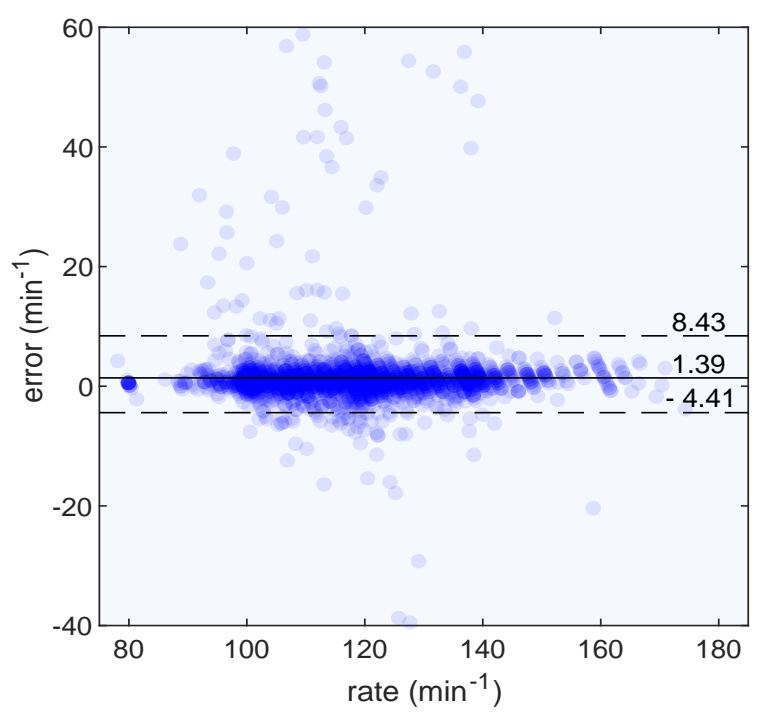

Figure 4. Blad-Altman plot of the $\mathrm{CC}$ rate error for one replica with $P_{\mathrm{th}}=26$. The black line shows the mean error, and the dashed black lines depict the $95 \%$ level of agreement.

rate absolute error of of $2.8 \mathrm{~min}^{-1}$.

This algorithm would permit giving feedback to the rescuer on the CC rate every $5 \mathrm{~s}$. Nevertheless, further analysis are required with complete PPG signals of larger datasets.

\section{Acknowledgements}

This work received financial support from the Spanish Ministerio de Economía y Competitividad, project TEC2015-64678-R, jointly with the Fondo Europeo de Desarrollo Regional (FEDER), from the University of the Basque Country via Ayuda a Grupos de Investigación GIU17/03, from the Basque Goverment through the grant PRE 2017 1 0112, and was also partially supported by NIH grant HL 077887 (AHÍ) and HL 077873.

\section{References}

[1] Atwood C, Eisenberg MS, Herlitz J, Rea TD. Incidence of EMS-treated out-of-hospital cardiac arrest in Europe. Resuscitation 2005;67(1):75-80.

[2] Nolan JP, Soar J, Zideman DA, Biarent D, Bossaert LL,
Deakin C, Koster RW, Wyllie J, Böttiger B. European resuscitation council guidelines for resuscitation 2010 section 1. Executive summary. Resuscitation 2010;81(10):12191276.

[3] Abella BS, Edelson DP, Kim S, Retzer E, Myklebust H, Barry AM, OHearn N, Hoek TLV, Becker LB. CPR quality improvement during in-hospital cardiac arrest using a real-time audiovisual feedback system. Resuscitation 2007; 73(1):54-61.

[4] Kramer-Johansen J, Myklebust H, Wik L, Fellows B, Svensson L, Sørebø H, Steen PA. Quality of out-of-hospital cardiopulmonary resuscitation with real time automated feedback: a prospective interventional study. Resuscitation 2006;71(3):283-292.

[5] Yeung J, Meeks R, Edelson D, Gao F, Soar J, Perkins GD. The use of CPR feedback/prompt devices during training and CPR performance: a systematic review. Resuscitation 2009;80(7):743-751.

[6] Gruber J, Stumpf D, Zapletal B, Neuhold S, Fischer H. Real-time feedback systems in CPR. Trends in Anaesthesia and Critical Care 2012;2(6):287-294.

[7] Tamura T, Maeda Y, Sekine M, Yoshida M. Wearable photoplethysmographic sensorspast and present. Electronics 2014;3(2):282-302.

[8] Elgendi M. On the analysis of fingertip photoplethysmogram signals. Current cardiology reviews 2012;8(1):14-25.

[9] Allen J. Photoplethysmography and its application in clinical physiological measurement. Physiological Measurement 2007;28(3):R1.

[10] Wijshoff RW, van der Sar T, Peeters WH, Bezemer R, Aelen P, Paulussen IW, Ordelman SC, Venema A, van Berkom PF, Aarts RM, et al. Detection of a spontaneous pulse in photoplethysmograms during automated cardiopulmonary resuscitation in a porcine model. Resuscitation 2013;84(11):1625-1632.

[11] Xu J, Li C, Zheng L, Han F, Li Y, Walline J, Fu Y, Yao $\mathrm{D}$, Zhang $\mathrm{X}$, Zhang $\mathrm{H}$, et al. Pulse oximetry: A noninvasive, novel marker for the quality of chest compressions in porcine models of cardiac arrest. PLoS ONE 2015; 10(10):e0139707.

Address for correspondence:

E. Aramendi

Engineering School of Bilbao

elisabete.aramendi@ehu.eus 